\title{
Studies of Small-Amplitude Red Variables
}

\author{
John R. Percy, Winnie Au, Adrien Desjardins and Lawrence Yu \\ Erindale College, University of Toronto, Mississauga, ON Canada L5L \\ $1 C 6$
}

\author{
AAVSO Photoelectric Photometry Program \\ 25 Birch St., Cambridge MA 02138-1205 USA
}

\begin{abstract}
We describe a survey of suspected small-amplitude red variables, and long-term (up to 10 years) monitoring of about two dozen such variables.
\end{abstract}

\section{Introduction}

Small-amplitude red variables (SARVs) are $\mathrm{M}$ giants and supergiants which are pulsating with small amplitudes (up to $2.5 \mathrm{mag}$ ) and with periods of 20 to 200 days or more. Most SARVs are bright stars. There are 164 known and 136 suspected SARVs among the approximately $500 \mathrm{M}$ giants and supergiants in the Bright Star Catalogue. SARVs are believed to be part of a sequence of red variables extending from the so-called $\sigma$ Librae stars with periods of 10 to 20 days, to the large-amplitude, long-period Mira stars. Many SARVs have a well-defined period, but the nature and cause of the irregularity (found in all of these stars) is not clear, nor is the pulsation mode. Many of these stars have a much longer secondary period, the nature of which is also not clear.

\section{Observations}

Standard photometric techniques were used, with a comparison and a check star for each variable. The internal scatter of the data ranges from 0.007 to slightly greater than 0.010 .

1. We are engaged in an ongoing survey of known and suspected SARVs using: (a) the $0.4 \mathrm{~m}$ teaching telescope of the University of Toronto; (b) the $0.25 \mathrm{~m}$ Automatic Photometric Telescope on Mt. Hopkins AZ (Percy \& Au 1994); (c) the AAVSO Photoelectric Photometry Program (Percy et al. 1994 and references therein).

2. Many SARVs have been monitored for up to a decade as part of the AAVSO's permanent photoelectric photometry program. The results presented in the table have been obtained by: (a) inspection of the light curves; (b) datecompensated discrete Fourier transform (Ferraz-Mello 1981) using a computer program kindly provided by E.P. Belserene; (c) autocorrelation analysis (Percy et al. 1993). 


\section{Results}

For the stars in the survey program, the incidence and amplitude of variability increase with later spectral type. No such correlation is apparent in the monitoring program, probably because the stars in this program have been chosen for their large amplitude. Most of the stars in the monitoring program show clear periodicity. W Boo appears to be a double-mode pulsator. At least half of the stars show a long period, typically an order of magnitude longer than the "short" period. There is no clear correlation between period and spectral type. Stars of the same spectral type can have "short" periods differing by an order of magnitude. This may be partly because of the complexity of the variability, or because of luminosity differences between the stars. Or it may be due to the excitation of different modes in different stars.

\begin{tabular}{|c|c|c|c|c|c|c|}
\hline Star & Sp T & & GCVS & & Resu & \\
\hline & & Type & Range & Period & $\Delta \mathrm{V}$ & Period(s) \\
\hline TV Psc & M3-4 III & SR & $0.77 \mathrm{~V}$ & 49.1 & 0.80 & 33.11, 1400: \\
\hline RZ Ari & M6 III & SRb & $0.39 \mathrm{~V}$ & 30 & 0.50 & $56: 500:$ \\
\hline$\rho$ Per & M4 II-III & SRb & $0.70 \mathrm{~V}$ & $50:$ & 0.40 & 135, long: \\
\hline CE Tau & M2 Iab-Ib & SRc & $0.31 \mathrm{~V}$ & 165 & 0.65 & 272,1200 \\
\hline$\alpha$ Ori & M1-2 Iab & SRC & $1.3 \mathrm{~V}$ & 2335 & 0.65 & $200:, 2000$ \\
\hline$\eta \mathrm{Gem}$ & M3 III & SRat & $0.75 \mathrm{~V}$ & 232.9 & 0.55 & 239.8 \\
\hline V614 Mon & $\mathrm{C} 4,5$ (R5) & $\mathrm{SRb}$ & $0.35 \mathrm{~V}$ & $60:$ & 0.45 & 80, long: \\
\hline RS Cnc & M6e Ib-IÍ & SRc: & $1.5 p$ & 120: & 1.40 & 228, long: \\
\hline IN Hya & M4 III & SRb & $0.60 \mathrm{~V}$ & 65: & 0.90 & $100-150:$ \\
\hline VY ÜMa & C6.3 (No) & & & - & 0.50 & 200:: \\
\hline VW UMa & & SR & $0.86 \mathrm{~V}$ & 610 & $0.5:$ & \\
\hline FS Com & M5 III & SRb & $0.8 \mathrm{~V}$ & 58: & 0.55 & 55.51, long: \\
\hline FP Vir & M4 III & SRb & $0.63 \mathrm{~V}$ & 40: & 0.80 & 70.1 , long: \\
\hline $\begin{array}{l}\text { W Boo } \\
\text { ST Her }\end{array}$ & $\begin{array}{l}\text { M2-4 III } \\
\text { M6-7 III }\end{array}$ & SRb: & $0.67 \mathrm{~V}$ & 450: & 0.40 & $24.71,56.67$ \\
\hline & M6-7 III & SRb & $1.5 p$ & 148.0 & 1.05 & 500: \\
\hline R Lyr & M5 III & SRb & $1.12 \mathrm{~V}$ & 46: & 0.60 & 53.41, long: \\
\hline EU Del & M6.4 III & $\mathrm{SRb}$ & $1.11 \mathrm{~V}$ & 59.7 & 0.95 & 62.74 \\
\hline U Del & M5 II-III & SRb & $1.3 \mathrm{~B}$ & 110: & 0.80 & 90,1000 \\
\hline W Cyg & M4-6e III & SRb & $2.1 \mathrm{~B}$ & 131.1 & 1.20 & 120:, 260: \\
\hline V1339 Cyg & M3-6 & SRb & $1.2 \mathrm{~V}$ & 35: & 0.40 & $50,800:$ \\
\hline$\mu \mathrm{Cep}$ & M2e Ia & SRc & $1.67 \mathrm{~V}$ & 730 & 0.75 & 850 \\
\hline$\chi \mathrm{Aqr}$ & M3 III & Lb & $0.16 \mathrm{~V}$ & - & 0.40 & $35,250:$ \\
\hline TX Psc & $\mathrm{C} 7,2$ (N0) & $\mathrm{Lb}$ & $0.41 \mathrm{~V}$ & - & 0.50 & 144 and/or 278 \\
\hline XZ Psc & M5 II & $\mathrm{Lb}$ & $0.36 \mathrm{~V}$ & - & 0.45 & 52.96 \\
\hline
\end{tabular}

Acknowledgments. We thank the AAVSO and its photoelectric observers for their participation, and NSERC Canada for a research grant.

\section{References}

Ferraz-Mello, S. 1981, AJ, 86, 619

Percy, J.R., \& Au, W. 1994, IAU IBVS No. 4114

Percy, J.R., et al. 1993, PASP, 105, 287

Percy, J.R., et al. 1994, PASP, 106, 61 